\title{
Recent Advances in the Photocatalytic Degradation of Direct Blue Dyes: A Critical Minireview and Analysis Study
}

\author{
Waleed Jadaa, Anand Prakash and Ajay K Ray* \\ Department of Chemical and Biochemical Engineering, Canada
}

*Corresponding author: Ajay K Ray, Department of Chemical and Biochemical Engineering, The University of Western Ontario, London, ON N6A 5B9, Canada

\section{ARTICLE INFO}

Received: 㗀 January 04, 2021

Published: 幽 January 28, 2021

Citation: Waleed Jadaa, Anand Prakash, Ajay K Ray. Recent Advances in the Photocatalytic Degradation of Direct Blue Dyes: A Critical Minireview and Analysis Study. Biomed J Sci \& Tech Res 33(3)-2021. BJSTR. MS.ID.005411.

Keywords: Direct Blue Dyes; Photocatalytic Degradation; Photocatalyst; Dopant; Kinetics

\begin{abstract}
Different dyes are used in numerous industries, including food, paper, ink, and the textile industry. Among various dye types, Direct Dyes have great significance because of their properties and applications. Such applications are paper, cotton, leather, and cellulose. The textile industry is considered a main contributor to the water pollution problem because it releases large quantities of coloured liquid waste. The majority of dyes and their products are considered carcinogenic, toxic, and resistant compounds. Therefore, the application of common wastewater treatments is not sufficient for dye removal such as adsorption, biological and chemical degradation. The advanced oxidation process using photocatalytic oxidation is an effective technique that can mineralize complicated aromatics and different organic dyes among various treatment methods. In this review, the photocatalytic degradation of Direct Blue dyes is discussed. The review is mainly focused on the recent advances in the photocatalysts and their activity improvement using different dopants.
\end{abstract}

\section{Mini Review}

In different things, including foods and various materials, gaining certain colours is generally obtained by adding substances widely known as dyes and pigments [1,2]. In the applied medium, water or oil, dyes are soluble materials primarily used for textile materials, whereas pigments are typically insoluble substances utilized via a dispersion method to obtain different products: inks and paints [1-4]. Different dyes are utilized for various product colorations in the textile industry, with an estimated amount between $7 \times 10^{5}$ and $7 \times 10^{7}$ tons worldwide annually [5,6]. The dye structure generally consists of two main constituents, namely, chromophore and auxochromes. The function of chromophores is light absorption to produce a certain colour. Hence, they are fundamentally responsible for the dye colour. Examples of chromophore groups are azo $(-\mathrm{N}=\mathrm{N}-)$, carbonyl $(\mathrm{C}=\mathrm{O})$ and methine $(-\mathrm{CH}=)$. Meanwhile, auxochromes have two different functions: playing a role in colour enhancement, more precisely, increase the colour intensity and a role in dye attachment to the surface of the applied medium, in the textile industry, attached dye to the fiber surface. In dyes, the auxochromes are classified into two main groups: acidic and basic groups.

While acidic auxochromes can include $-\mathrm{OH},-\mathrm{COOH}$, and $-\mathrm{SO} 3 \mathrm{H}$, the basic groups of auxochromes involve $-\mathrm{NH}_{2}, \mathrm{NHR}$, and $-\mathrm{NR}_{2}$ [1$3,7-8]$. Dyes are categorized into various classes depending on different aspects. However, the classification based on their usage and chemical structure are the most popular ways. Depending on the type of applied method to the used substrate, dyes are categorized into different groups. Such groups include Acid Dyes, Basic Dyes, Direct Dyes, Reactive Dyes, Disperse Dyes, Solvent Dyes, Sulfur Dyes, Vat Dyes and Mordant Dyes [3,8]. Among different dye types, Direct Dyes have great importance due to both their characteristics and applications. In terms of their applications, Direct Dyes are applied with no requirements for the fixing process, precisely without the step of affixing agent. This leads to a simplified dyeing process and lowered process operating costs. Their applications include paper, 
cotton, leather, cellulose, and nylon. These dyes are typically watersoluble with a strong affinity to cellulose fibers. Their solubility is enhanced due to the presence of sulfonate groups in some types.

Though such substances are anionic dyes, their categorization is not under the acid dyes class. This is because the attachment to the fiber surface does not usually occur by acid groups [8-11]. The textile industry waste is a major contributor to aquatic life pollution among different waste types due to releasing a huge amount of coloured wastewater to the environment. The reason behind such significant wastewater amounts is the features of the dyeing process itself. The dye bath tank's draining and cleaning after each dyeing process are the two main factors responsible for releasing coloured wastewater [12]. As the dye's usage is dramatically increased due to their numerous applications, the effluent of their usage, especially textile industries, is considered a big challenge in the wastewater treatment area. This is because of various factors: high levels of Biochemical Oxygen Demand (BOD) and Chemical Oxygen Demand (COD) $[13,14]$; most dyes and their by-products are carcinogenic and highly toxic compounds [15-19] and difficult to be completely degraded because they are more resistant substances for the typical water treatment methods such as adsorption, photolysis, biological and chemical degradation [20-22].

Among different alternative treatments, applying the Advanced Oxidation Process (AOP) using the photocatalytic oxidation approach is a promising method for resistant materials such as complicated aromatics and organic dyes. The use of the photodegradation technique leads to degrading highly toxic compounds to environmentally friendly minerals [3,20,23-26]. Therefore, this way has been attracted many researchers. This minireview paper addresses the application of AOP using the photocatalytic degradation approach for Direct Blue dyes. This paper's main focus is discussing the recent advances in photocatalytic degradation in terms of using different photocatalysts and their modifications using various dopants.

\section{Literature Review}

In terms of Direct Blue dyes, El-Bahy, et al. [27] examined the photocatalytic degradation of Direct Blue dye (DB53) using various lanthanide ions doped with $\mathrm{TiO}_{2}$. Under UV irradiation, the study demonstrated that the type of dopant has a high degree of impact on the catalyst surface properties such as texture structure, particle size and bandgap. $\mathrm{Gd}_{-}-\mathrm{TiO}_{2}$ appeared to be the most effective catalyst, resulting in the highest dye removal due to its excellent surface properties. Using a $\mathrm{Cu}_{2} \mathrm{O}$ catalyst, it was observed that the hydrothermal temperature has a key role in determining the main properties of the synthesized catalyst, including the bandgap, shape, and surface area. In terms of DB53 degradation, the catalyst with a nanorod shape was highly effective compared to other catalyst shapes [28]. In contrast, Mohamed, et al. [29] changed the hydrothermal time for preparing $\mathrm{YVO}_{4}$ nanoparticles from $4 \mathrm{~h}$ to 24 h. Their study found that the $\mathrm{YVO}_{4}$ nanoparticles with a size of 11 $\mathrm{nm}$ exhibit the optimum behaviour of catalyst activity. Sobana, et al. [30] applied 2\% Ag-doped $\mathrm{TiO}_{2}$ for the degradation of DB53 using both UV and solar light irradiation.

The application of solar irradiation for the Ag-doped $\mathrm{TiO}_{2}$ registered a higher DB53 degradation than UV light utilization. Sobana, et al. [31] concluded that the catalyst activated carbon AC-ZnO has a good activity for DB53 removal under solar light irradiation. While alkaline $\mathrm{pH}$ appeared to be more preferable than acidic $\mathrm{pH}$, catalyst grinding showed a negative impact on DB53 degradation efficiency. Mohamed, et al. [32] observed that the energy band gap of $\mathrm{Pt} / \mathrm{Ti}$-Na-mordenite catalyst proportionally decreases when the amount of Pt is increased. The doping of Pt at high concentration ( $0.4 \mathrm{wt} . \%)$ had no significant impact on energy bandgap reduction. Under visible light irradiation, 100\% DB53 dye removal was registered at a Pt concentration of 0.3 wt.\% after 60 min irradiation, resulting in the highest degradation. Sobana, et al. [33] used Ag-doped $\mathrm{TiO}_{2}$ for the degradation of DB53 aqueous solution. Under UVA irradiation, the study outcomes proved that the photocatalyst activity increases as the dopant concentration is increased. Ag particles on the catalyst surface work as electron traps, resulting in a significant enhancement of electron-hole separation and resulting in the reduction of recombination rate.

In terms of reaction rate, the obtained results showed that DB53 dye degradation is described by pseudo-first order kinetics using different catalyst types [29-32]. DB71 was degraded under UVC irradiation. The findings suggested that high dye removal is achieved for two different cases: UV irradiation and UV/ $\mathrm{TiO}_{2}$. In terms of $\mathrm{pH}$ solution and temperature, there was little effect on DB71 degradation Saien, et al. [34,35] found that the prepared catalyst $\mathrm{CuO}-\mathrm{ZnO}$ has a significantly higher photoactivity compared to reagent-grade $\mathrm{ZnO}$ under visible light irradiation. The nanocomposite with (1:3) CuO-ZnO was the most successful application due to obtaining the highest DB71 removal. Habibi, et al. [36] demonstrated that the efficiency of DB71 removal reached $82 \%$ using a bi-component nanocomposite that consists of cobalt metatitanate and cobalt oxide. In the presence of $\mathrm{K}_{7}\left[\mathrm{PMo}_{2} \mathrm{~W}_{9} \mathrm{O}_{39}\right] \cdot 19 \mathrm{H}_{2} \mathrm{O}$ and $\mathrm{H}_{2} \mathrm{O}_{2}$, the DB71 removal rate registered $65 \%$ for $60 \mathrm{~min}$. The optimal conditions of DB71 removal were 0.6 $\mathrm{g} / \mathrm{L}, 0.08 \mathrm{~mol} / \mathrm{L}$, and $90 \mathrm{~min}$ for catalyst loading, $\mathrm{H}_{2} \mathrm{O}_{2}$, and reaction time, respectively. Under such conditions, the percentage of DB71 degradation was approximately $87 \%$ Tabatabaee, et al. [37,38] tested the ability of $\mathrm{CuFeO}_{2} / \mathrm{ZnO}$ as a photocatalyst for degradation of DB71.

The study demonstrated that the dye mineralization is confirmed by TOC measurements at natural $\mathrm{pH}$ under visible light. Pugazhenthiran, et al. [39] discussed the possibility of Au$\mathrm{Nx}-\mathrm{TiO}_{2}$ and $\mathrm{Au}-\mathrm{TiO}_{2}$ nanospheres application in the degradation of DB71. Under solar irradiation, the obtained results showed that the synthesized catalysts exhibit a superior photoactivity performance. In terms of catalyst stability, the prepared catalysts 
had no photoactivity reduction even after eight consecutive tests. Sathishkumar, et al. [40] aimed to increase ZnO photoactivity by doping $\mathrm{CoFe}_{2} \mathrm{O}_{4}$ on its surface. Under visible light irradiation, the activity synthesized catalyst was examined for DB71 degradation using a $150 \mathrm{~W}$ lamp with a wavelength $(\lambda \geq 400 \mathrm{~nm})$. The study findings manifested a reduction of optical bandgap energy. In the presence of both used catalyst and PMS, the highest value of DB71 degradation rate was achieved. Interestingly, a power-law model is registered for the DB71 degradation kinetics. Though the degradation rate was expressed as a sum of two terms: photolysis and photocatalysis, the individual terms had a power order with a value of 1.83 and 2.16, respectively Saien, et al. [34].

In contrast, the obtained data of DB71 degradation was accurately fitted by pseudo-first order reaction kinetics Boumaza, et al. [38] As a part of their study, Habibi, et al. [41] investigated the degradation of DB160 solution using commercial TiO2 catalyst. With a UV source of $400 \mathrm{~W}$, the obtained results found that complete decolorization is successfully achieved. With respect to the reaction kinetics, pseudo-first order kinetics clearly described the DB160 degradation. It was also observed that inorganic ions have an inhibitory effect on the DB160 degradation rate Saroj, et al. [42] tested different concentrations of Fe-doped $\mathrm{TiO} 2\left(\mathrm{Ti}_{1-\mathrm{x}} \mathrm{Fe}_{\mathrm{x}} \mathrm{O}_{2}\right)$. Two different irradiation methods, namely, UV light and direct sunlight, were applied for DB199 degradation within real industry wastewater. The results revealed that the catalyst $\mathrm{Ti}_{0.96} \mathrm{Fe}_{0.04} \mathrm{O}_{2}$ has the highest photoactivity in both reactor types, indicating that the reactor type has no influence on the catalyst activity. The DB129 degradation was examined by applying the ZnO catalyst. By applying the sol-gel method, $\mathrm{ZnO}$ nanoparticles were prepared using Arabic gum (AG) as a template. Under visible light, it was observed that using $\mathrm{ZnO}$ nanoparticles leads to a high dye removal up to $95 \%$ Fardood, et al [43].

With respect to DB15 dye, Lamba, et al. [44] synthesized $\mathrm{CeO}_{2}$ ZnO nanodisks using a precipitation method. In a double-walled reactor, an aqueous solution of $50 \mathrm{mg} / \mathrm{L}$ DB15 was irradiated using natural solar light for $4 \mathrm{~h}$ during the time between 10 am to 2 $\mathrm{pm}$. Their study found that the $\mathrm{CeO}_{2}-\mathrm{ZnO}$ catalyst shows excellent activity that could possibly be due to an enhanced recombination rate Ebrahimi, et al. [45] discussed the impact of transition metal doped $\mathrm{ZnO}$ nanoparticles on the photodegradation of DB 15 using both UV and visible light irradiation. The synthesized $\mathrm{ZnO}$ was doped with three different transition metals: $\mathrm{Ag}, \mathrm{Cu}$, and $\mathrm{Mn}$. The findings demonstrated that the transition metal-doped catalyst has higher activity in comparison with undoped ZnO. For UV irradiation, the optimum dye removal was 74\%, registered for Ag-doped $\mathrm{ZnO}$. In contrast, the $\mathrm{Cu}$-doped $\mathrm{ZnO}$ had the highest degradation rate with a value of $70 \%$ under visible light irradiation. The influence of catalyst morphology on its photocatalytic activity was investigated by Jo, et al. [46] Among different morphologies, the catalyst with nanoflower morphology registered the highest photoactivity.
Depending on the surface morphology, the DB15 degradation had the following order: nanoflowers $>$ nanotubes $>$ nanospheres Deng, et al. [47] increased the surface area of Fe-based metallic glass ribbons using etched porous ribbons and evaluated for DB15 degradation. The outcomes revealed that the application of etching treatment in $20 \% \mathrm{HF}$ solution for 40 min leads to a big improvement in the catalytic activity due to obtaining higher rates of DB15 degradation. Zhan, et al. [48] synthesized Copper hydroxide nitrate $\left(\mathrm{Cu}_{2}(\mathrm{OH})_{3} \mathrm{NO}_{3}\right)$ by a solvothermal method using anhydrous ethanol. The synthesized catalyst appeared to have a complex structure with spherical morphology evaluated for DB15 degradation. The catalyst registered a high activity in the DB15 removal compared with the catalyst prepared by the typical reaction of $\mathrm{NaOH}$ and $\mathrm{Cu}\left(\mathrm{NO}_{3}\right)_{2}$ in an aqueous solution.

\section{Conclusion}

While scanning the relevant literature of Direct Blue dyes, the main conclusions are as follows:

1. The majority of previous studies have been mainly focused on two dyes, namely: DB53 and DB71. Meanwhile, few studies have been registered for the evaluation of DB15 photodegradation.

2. Different dopants have been applied for $\mathrm{TiO} 2$ or $\mathrm{ZnO}$ photocatalyst modification. It is observed that the dopant concentration has an impact on the photocatalyst performance. However, the higher dopant concentrations have no effect on the catalyst photoactivity.

3. The dopant type influences the photocatalyst surface characteristics such as surface texture, particle size and energy bandgap.

4. With different photocatalysts, the photodegradation of Direct Blue dyes is well described by pseudo-first order kinetics.

\section{References}

1. Erkurt HA (2010) Biodegradation of azo dyes. Springer.

2. Sharma SK, Sanghi R (Eds.). (2012). Advances in water treatment and pollution prevention. Springer Science \& Business Media.

3. Benkhaya S, Mrabet S, El Harfi A (2020) A review on classifications, recent synthesis and applications of textile dyes. Inorg Chem Commun pp. 107891.

4. Vázquez Ortega F, Lagunes I, Trigos Á (2020) Cosmetic dyes as potential photosensitizers of singlet oxygen generation. Dyes and Pig 176: 108248.

5. Chandanshive VV, Kadam SK, Khandare RV, Kurade MB, Jeon BH, et al. (2018) In situ phytoremediation of dyes from textile wastewater using garden ornamental plants, effect on soil quality and plant growth. Chemo 210: 968-976.

6. Wang CC, Li JR, Lv XL, Zhang YQ Guo G, et al. (2014) Photocatalytic organic pollutants degradation in metal-organic frameworks. Energ \& Environ Sci 7(9): 2831-2867.

7. Jie RH, Guo GB, Zhao WG, An SL (2013) Preparation and photocatalytic degradation of methyl orange of nano-powder TiO2 by hydrothermal method supported on activated carbon. J Synth Cryst (42): 2144-2149. 
8. Muhd Julkapli N, Bagheri S, Bee Abd Hamid S (2014) Recent advances in heterogeneous photocatalytic decolorization of synthetic dyes. The Sci World J.

9. Khatri A, Peerzada MH, Mohsin M, White M (2015) A review on developments in dyeing cotton fabrics with reactive dyes for reducing effluent pollution. J Clean Prod 87: 50-57.

10. Chen X, Ding K, Jun L (2015) Synthesis, identification and application of aldehyde reactive dyes. Dyes Pig 123: 404-412.

11. Cavaco Paulo A, Morgado J, Almeida L, Kilburn D (1998) Indigo backstaining during cellulase washing. Text Res J 68(6): 398-401.

12. Markandeya S, Shukla P, Mohan D (2017) Toxicity of disperse dyes and its removal from wastewater using various adsorbents: a review. Res J Envir Toxi 11: 72-89.

13. Bansal P, Sud D (2012) Photodegradation of commercial dye, C Reactive Blue 160 using ZnO nanopowder: Degradation pathway and identification of intermediates by GC/MS. Sep puri techn 85: 112-119.

14. Champagne PP, Nesheim ME, Ramsay JA (2010) Effect of a non-ionic surfactant, Merpol, on dye decolorization of Reactive blue 19 by laccase. Enzy micro techn 46(2): 147-152.

15. Hsiao YC, Wu TF, Wang YS, Hu CC, Huang C, et al. (2014) Evaluating the sensitizing effect on the photocatalytic decoloration of dyes using anatase-TiO2. App Cat B: Envir 148: 250-257.

16. Zhang S (2014) Preparation of controlled-shape ZnS microcrystals and photocatalytic property. Ceram inter 40(3): 4553-4557.

17. Rosa JM, Garcia VS, Boiani NF, Melo CG, Pereira MC, et al. (2019) Toxicity and environmental impacts approached in the dyeing of polyamide, polyester and cotton knits. J Envir Chem Eng 7(2): 102973.

18. Bouberka Z, Benabbou KA, Khenifi A, Maschke U (2014) Degradation by irradiation of an Acid Orange 7 on colloidal TiO2/(LDHs). J Photochem and Photobio A: Chem 275: 21-29.

19. Pant B, Pant HR, Barakat NA, Park M, Han TH, et al. (2014) Incorporation of cadmium sulfide nanoparticles on the cadmium titanate nanofibers for enhanced organic dye degradation and hydrogen release. Ceram Inter 40(1): 1553-1559.

20. Javaid R, Qazi UY (2019) Catalytic oxidation process for the degradation of synthetic dyes: an overview. Inter J Envir Res Pub Hea 16(11): 2066.

21. Nenavathu BP, Rao AK, Goyal A, Kapoor A, Dutta RK, et al. (2013) Synthesis, characterization and enhanced photocatalytic degradation efficiency of Se doped $\mathrm{ZnO}$ nanoparticles using trypan blue as a model dye. App Cat A: Gen 459: 106-113.

22. Huo Y, Xie Z, Wang X, Li H, Hoang M, et al. (2013) Methyl orange removal by combined visible-light photocatalysis and membrane distillation. Dyes Pig 98(1): 106-112.

23. Youssef Z, Colombeau L, Yesmurzayeva N, Baros F, Vanderesse R, et al. (2018) Dye-sensitized nanoparticles for heterogeneous photocatalysis: Cases studies with $\mathrm{TiO} 2, \mathrm{ZnO}$, fullerene and graphene for water purification. Dyes Pig 159: 49-71.

24. Sleiman M, Vildozo D, Ferronato C, Chovelon JM (2007) Photocatalytic degradation of azo dye Metanil Yellow: optimization and kinetic modeling using a chemometric approach. App Cat B: Envir 77(1-2): 1-11.

25. Li R, Song X, Huang Y, Fang Y, Jia M, et al. (2016) Visible-light photocatalytic degradation of azo dyes in water by Ag3P04: An unusual dependency between adsorption and the degradation rate on $\mathrm{pH}$ value. J Mole Cat A: Chem 421: 57-65.

26. Karamat N, Ehsan MF, Ashiq MN, Ijaz S, Najam ulHaq M, et al. (2019) Synthesis, characterization and photocatalytic activity of LaNdZr207 supported SnSe nanocomposites for the degradation of Foron blue dye. App Sur Sci 463: 1019-1027.
27. El Bahy ZM, Ismail AA, Mohamed RM (2009) Enhancement of titania by doping rare earth for photodegradation of organic dye (Direct Blue). J Haz Mat 166(1): 138-143.

28. Aazam ES, Mohamed RM (2013) Environmental remediation of Direct Blue dye solutions by photocatalytic oxidation with cuprous oxide. J All Comp 577: 550-555.

29. Mohamed RM, Harraz FA, Mkhalid IA (2012) Hydrothermal synthesis of size-controllable Yttrium Orthovanadate (YVO4) nanoparticles and its application in photocatalytic degradation of direct blue dye. J All Comp 532: 55-60

30. Sobana N, Subash B, Swaminathan M (2015) Effect of operational parameters on photodegradation of Direct Blue 53 by silver loadedtitania under ultraviolet and solar illumination. Mat Sci Semic Proc 36: 149-155.

31. Sobana N, Swaminathan M (2007) Combination effect of ZnO and activated carbon for solar assisted photocatalytic degradation of Direct Blue 53. Sol Energ Mat Sol Cel 91(8): 727-734.

32. Mohamed RM, Baeissa ES (2013) Mordenite encapsulated with Pt-TiO2: Characterization and applications for photocatalytic degradation of direct blue dye. J All Comp 558: 68-72.

33. Sobana N, Muruganadham M, Swaminathan M (2006) Nano-Ag particles doped TiO2 for efficient photodegradation of direct azo dyes. J Mole Cat A: Chem 258(1-2): 124-132.

34. Saien J, Soleymani AR (2007) Degradation and mineralization of Direct Blue 71 in a circulating upflow reactor by UV/TiO2 process and employing a new method in kinetic study. J Haz Mat 144(1-2): 506-512.

35. Salehi K, Bahmani A, Shahmoradi B, Pordel MA, Kohzadi S, et al. (2017) Response surface methodology (RSM) optimization approach for degradation of Direct Blue 71 dye using CuO-ZnO nanocomposite. Inter J Envir Sci Tech 14(10): 2067-2076.

36. Habibi MH, Shojaee E (2017) Bi-component cobalt metatitanate and cobalt oxide nano-composite for high efficient photocatalytic degradation of triazo Direct Blue 71: synthesis, characterization and surface properties. J Mat Sci: Mat Electr 28(15): 11013-11019.

37. Tabatabaee M, Roozbeh M, Roozbeh M (2011) Catalytic effect of lucunary heteropolyanion containing molybdenum and tungsten atoms on decolorization of direct blue 71. Chine Chem Let 22(12): 1501-1504.

38. Boumaza S, Kaouah F, Hamane D, Trari M, Omeiri S, et al. (2014) Visible light assisted decolorization of azo dyes: Direct Red 16 and Direct Blue 71 in aqueous solution on the $\mathrm{p}-\mathrm{CuFeO}_{2} / \mathrm{n}-\mathrm{ZnO}$ system. J Mole Cat $\mathrm{A}$ : Chem 393: 156-165.

39. Pugazhenthiran N, Mangalaraja RV, Sathishkumar P, Murugesan S, Muneeswaran T, et al. (2018) Green synthesis of porous $\mathrm{Au}-\mathrm{N}$ x-TiO2 nanospheres for solar light induced photocatalytic degradation of diazo and triazo dyes and their eco-toxic effects. New J Chem 42(23): $18717-$ 18728.

40. Sathishkumar P, Pugazhenthiran N, Mangalaraja RV, Asiri AM, Anandan $\mathrm{S}$, et al. (2013) $\mathrm{ZnO}$ supported $\mathrm{CoFe}_{2} \mathrm{O}_{4}$ nanophotocatalysts for the mineralization of Direct Blue 71 in aqueous environments. J Haz Mat 252: $171-179$

41. Habibi MH, Hassanzadeh A, Mahdavi S (2005) The effect of operational parameters on the photocatalytic degradation of three textile azo dyes in aqueous TiO2 suspensions. J Photoch Photobio A: Chem 172(1): 89-96.

42. Saroj S, Singh L, Singh SV (2019) Photodegradation of Direct Blue-199 in carpet industry wastewater using iron-doped TiO2 nanoparticles and regenerated photocatalyst. Inter J Chem Kin 51(3): 189-205.

43. Fardood ST, Ramazani A, Moradi S, Asiabi PA (2017) Green synthesis of zinc oxide nanoparticles using arabic gum and photocatalytic degradation of direct blue 129 dye under visible light. J Mat Sci: Mat Elect 28(18): 13596-13601. 
44. Lamba R, Umar A, Mehta SK, Kansal SK (2015) CeO2ZnO hexagonal nanodisks: efficient material for the degradation of direct blue 15 dye and its simulated dye bath effluent under solar light. J All Comp 620: 67-73.

45. Ebrahimi R, Hossienzadeh K, Maleki A, Ghanbari R, Rezaee R, et al. (2019) Effects of doping zinc oxide nanoparticles with transition metals (Ag, $\mathrm{Cu}, \mathrm{Mn}$ ) on photocatalytic degradation of Direct Blue 15 dye under UV and visible light irradiation. J Envir Hea Sci Eng 17(1): 1-14.

46. Jo WK, Tayade RJ (2016) Direct blue dye degradation using titanium nanostructures under energy-efficient UV-LED irradiation. J Mat Eng Perf 25(1): 83-90.

ISSN: 2574-1241

DOI: $10.26717 / B J S T R .2021 .33 .005411$

Ajay K Ray. Biomed J Sci \& Tech Res

(C) This work is licensed under Creative

Submission Link: https://biomedres.us/submit-manuscript.php
47. Deng Z, Zhang XH, Chan KC, Liu L, Li T (2017) Fe-based metallic glass catalyst with nanoporous surface for azo dye degradation. Chemo 174 76-81.

48. Zhan Y, Zhou X, Fu B, Chen Y (2011) Catalytic wet peroxide oxidation of azo dye (Direct Blue 15) using solvothermally synthesized copper hydroxide nitrate as catalyst. J Haz Mat 187(1-3): 348-354.

$\begin{array}{ll}\text { BIOMEDICAL } & \text { Assets of Publishing with us } \\ \text { RESEARCHES } & \text { - Global archiving of articles } \\ \text { - Immediate, unrestricted online access } & \text { - Rigorous Peer Review Process } \\ & \text { - Authors Retain Copyrights } \\ & \end{array}$

